\title{
DÉVERSOIRS D'ÉTANGS
}

\author{
Par M. R. PONROY \\ Ingénicur agronome, \\ Membre du Bureau du Syndicat.
}

Un déversoir d'étang doit :

$I^{\circ}$ Avoir une très grande puissance d'écoulcment;

$2^{\circ}$ S'opposer à la remontéc des poissons indésirables;

$3^{\circ}$ Etre de construction économique et d'entretien facile.

\section{I. - Puissance d'écoulement.}

Par année pluvieuse, comme par année de sécheresse, les déversoirs à grande puissance sont précieux.

Le déversoir doit pouvoir suffire à assurer, seul et sans le secours des bondes, l'écoulement des eaux reçues en excès, même par les plus grandes crues.

Il est, en outre, avantageux de pouvoir retenir dans un étang une plus grande quantité d'eau qui permettra de traverser les années de sécheresse sans perdre le poisson. Un déversoir à grande puissance permet de maintenir plus haut le niveau de l'étang, augmentant ainsi le volume d'eau et la surface utile sans augmenter la surface novée en temps de crues.

La première besogne, avant de commencer la construction d'un déversoir, est d'évaluer la largeur I qu'il faudra donner à ce déversoir pour Iui assurer un débit suffisant.

Il importe d'abord d'apprécier la surface du bassin d'alimentation de l'étang. Ce bassin, dans son ensemble, affecte toujours la forme d'un triangle plus ou moins vaste, le déversoir est à l'angle du triangle par lequel l'eau s'écoule.

En Sologne, on peut évaluer L par la formule suivante :

$$
\text { I. (en mètres) }=6 \times \frac{\mathrm{S} \text { (en Hectares) }}{\mathrm{s} \text { (en Hectares) } \times \mathrm{R} \text { (en hectomètres) }}
$$

ou : $\mathrm{L}=$ largeur du déversoir exprimée en mètres, la crête étant supposée constituée par une bordure de ciment de ro centimètres d'épaisseur.

$\mathrm{S}=$ surface du triangle constituant le bassin d'alimentation de l'étang, exprimée en Hectares, les surfaces plantées en pins ou autres bois peuvent en être retranchées, parce qu'elles retiennent suffisamment l'eau pour retarder l'effet de crue qu'elle pourrait avoir.

$\mathrm{s}=$ surface en eau de l'étang lorsqu'il est à son niveau, exprimée en Hectares.

$\mathrm{R}=$ longueur en hectomètres du bassin d'alimentation de l'étang. 
Exemple : un étang de 5 Hectares a un bassin de rxo Hectares long de 3 kilomètres. Son déversoir devra avoir une largeur de :

$$
\mathrm{I}=6 \times \frac{2 \mathrm{ro}}{5 \times 30}=8^{\mathrm{m}} 40
$$

Cette largeur peut paraître excessive, mais, en réalité, elle est prudente et pratique.

Le r/4 Août I 932 , il est tombé 43 millimètres d'eau en 12 heures. Si la terre avait été saturée d'eau lorsque cette pluie est arrivée, la crue eût été formidable. C'est co qui s'est produit en 19Jo, année qui vil tant d'étangs cmporter leurs chaussées, et fit perdre lant de poissons.

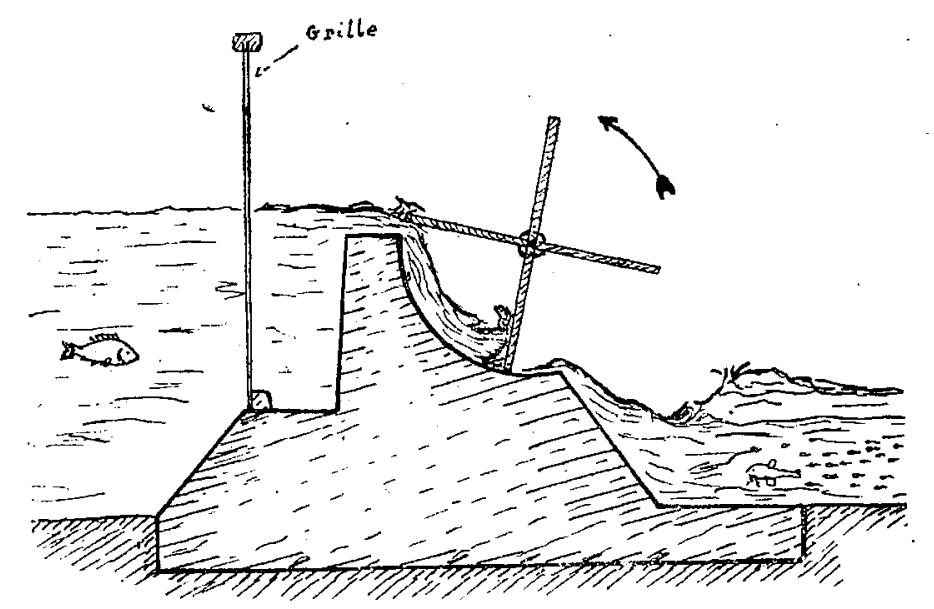

Fra. 13. - Roue à aubes installée sur un déversoir pour interdire la remontéc aux poissons indésirables.

\section{II. - Obstacle à la remontée des poissons indésirables.}

De même que les mauvaises herbes nuisent au blé, les mauvais poissons nuisent aux bons.

Pour se débarrasser de la vermine des Poissons-chats, Perches arc-enciel, Epinoches, etc. il ne suffit pas d'en débarrasser l'étang, il faut encore éviler une nouvelle invasion par la voie du déversoir.

Les grilles sont insuffisantes, ou alors elles s'encrassent continuellement.

La chute d'eau d'une hauteur supérieure à I mètre est la meilleure barrière.

Dans le cas où on ne peut disposer d'une chute de cette hauteur on peut avoir recours à une roue à aubes dont les palettes balajent le fond d'une auge, rendant ainsi toutes remontées impossibles (fig. r3).

Ce système peut aussi être avantageusement employé aux queues d'étang pour empêcher le poisson de quitter l'étang.

Mais c'est surtout dans les fossés où on ne peut établir de chute que ce 


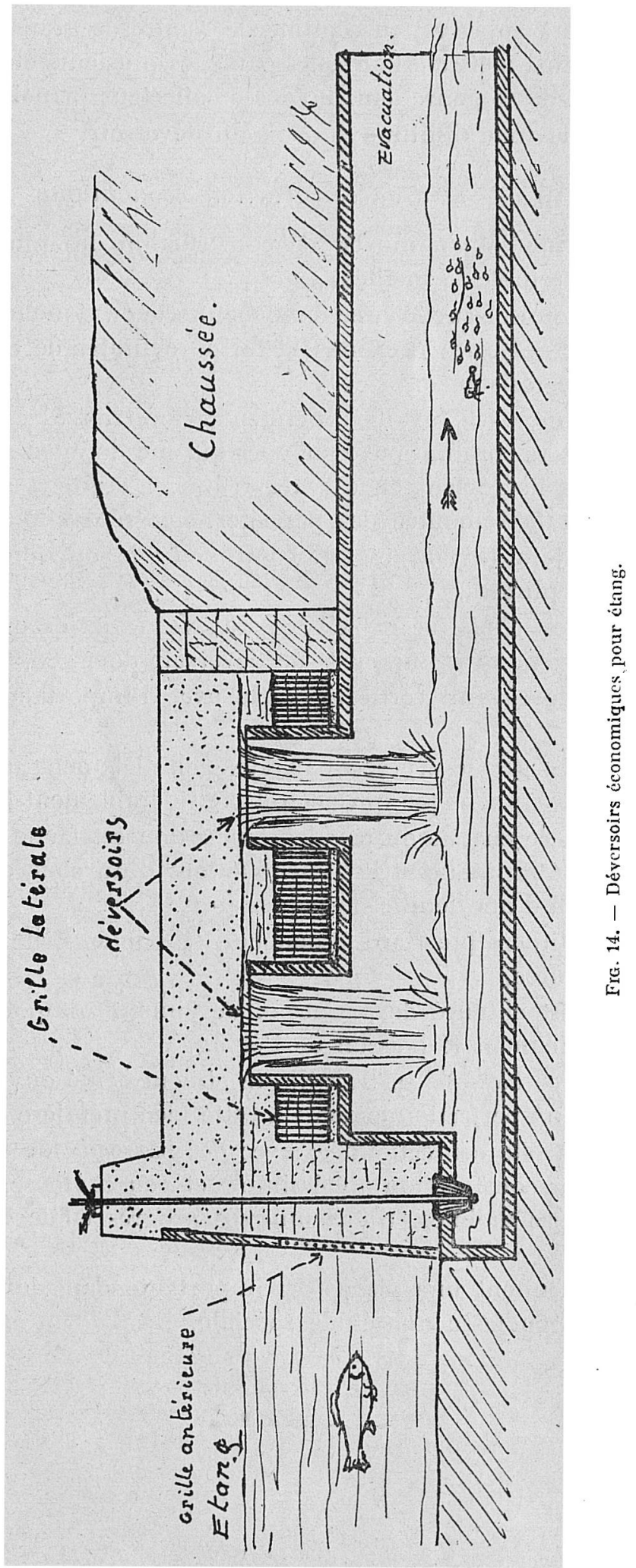


système de rouc à aubes est susceptible de rendre de grands services. On peut, en effet, ainsi mettre à l'abri des parasiles plusieurs étangs en installant un barrage de ce genre dans un fossé collecteur principal.

A noter que la roue diminue le débit du déversoir.

$$
\text { III. - Elre de construction économique. }
$$

Le ciment armé est un moyen de construction des plus intéressants. C'est celui qui semble le meilleur.

On peut économiser sur le cube de maçonnerie en donnant aux surfaces qui ont à subir de fortes pressions la forme cylindrique qui leur assure le maximum de résistance.

J'ai pu réaliser, avec tuyaux de ciment armé trouvés chez un cimentier, des déversoirs combinés avec bondes qui me donnent complète satisfaction. Le prix de revient en est très réduit. L'écoulement se lait avec chute entravant la remontée des parasites. Les réparations sont faciles.

Le croquis ci-joint (fig. ili) on fera comprendre le fonctionnement mieux que de longues phrases.

Le déversoir est constitué par des tuyaux verticaux de $0^{\mathrm{m}} 60$ de diamètre, ce qui correspond à une crète de déversoir de $\mathrm{I}^{\mathrm{m}} 8 \mathrm{o}$.

Le nombre de luyaux verticaux varie avec l'importance de l'écoulement à assurer.

En cas de très grosse crue, ces tuyaux complètement noyés fonctionnent comme des siphons, ce qui accroì considérablement leur débit.

La bonde placée en tète du canal d'évacuation est facile à construire à très bon marché en moulant le ciment autour d'un cône de bois préalablement entouré d'unc feuille de papier gras.

Ce cône constituera plus tard le bouchon de bonde. L'étanchẻité la plus parfaite est assurée.

L'ensemble est entouré de panneaux de ciment comportant des baies grillées qui retiennent le poisson de l'étang.

La facilité avec laquelle se travaille le ciment armé ouvre des possibililés de nombreux perfectionnements à cette idée première.

Les grilles ont été confectionnées avec des barreaux de fer noyés dans du ciment. Leur construction a élé simple el peu cô̂teuse, mais les barreaux ne sont pas mobiles. Il y a un gros progrès facile à faire dans ce sens.

Les grilles tiennent une place frès importante dans les étangs; elles méritent qu'on en fasse une élude spéciale. 